\title{
Effective viscosity of microswimmer suspensions
}

\author{
Salima Rafaï, Levan Jibuti, Philippe Peyla \\ Laboratoire de Spectrométrie Physique, Grenoble, France- UJF-CNRS UMR5588
}

\begin{abstract}
The measurement of a quantitative and macroscopic parameter to estimate the global motility of a large population of swimming biological cells is a challenge Experiments on the rheology of active suspensions have been performed. Effective viscosity of sheared suspensions of live unicellular motile micro-algae (Chlamydomonas Reinhardtii) is far greater than for suspensions containing the same volume fraction of dead cells and suspensions show shear thinning behaviour. We relate these macroscopic measurements to the orientation of individual swimming cells under flow and discuss our results in the light of several existing models.
\end{abstract}

PACS numbers: 47.63.-b, 47.57.-s, 47.50.-d

In nature, organisms that can propel themselves in a fluid medium are ubiquitous. While larger organisms, such as fish, use inertia in their motion, micro-organisms like spermatozoa, micro-algae or bacteria, move at low Reynolds number, where viscous forces dominate over the effects of inertia. A recent and currently unresolved issue involves understanding the hydrodynamics associated with the individual or collective motion of microswimmers through their fluid-mediated interactions [1]. Microswimmer suspensions have been shown to lead to complex dynamics such as the so-called weak turbulence or bioconvection phenomenon 2-4]. Such active suspensions are made of self-propelled particles that create a force multipole either at the front of the body, in which case they are called pullers, or at the back, in which case, they are pushers. The flow induced by the force multipole is responsible for hydrodynamic interactions which are expected to have a dramatic effect on dynamics and particularly on the rheology of the suspension.

Recently, theoretical efforts have been made to model the effective viscosity of active suspensions. Stokesian dynamic simulations by Ishikawa and Pedley [5] show a difference in effective viscosity for suspensions of swimming spherical 'squirmers' in a gravity field. Haines et al. 6] analytically showed that swimming results in a change in viscosity if the orientation distribution of swimmers is assumed to be anisotropic. More recently, Saintillan [7] used a simple kinetic model to study the rheology of a dilute suspension of self-propelled particles in a shear flow. He showed that suspensions of pullers exhibit increased effective viscosity compared to passive suspensions, while pusher suspensions exhibit a significant decrease in viscosity due to the motile activity; these results are consistent with previous predictions of Hatwalne et al. [8] who used coarse-grained active hydrodynamics to predict the rheology of active suspensions. Depending on swimmer types (puller or pusher), cell shape (spherical or ellipsoidal) and locomotion mechanisms, models can lead to very diverse results in terms of rheology. In order to better understand the effects of motility on viscosity, experimental work addressing the rheology of microswimmer suspensions is necessary.
Very recently, Sokolov and Aranson [9] measured the microrheology of suspensions of pusher-like bacteria. They found, as predicted by [7, 8], that the effective viscosity of such active suspensions decreases in comparison to passive particles at the same volume fraction. To our knowledge, no previous experimental data exist for puller-type micro-swimmer suspensions for which an increase of effective viscosity is predicted [7, 8]. This paper presents the first direct experimental macroscopic measurement of the effective viscosity of puller type microswimmer suspensions: Chlamydomonas Reinhardtii $(\mathrm{CR})$, a $10 \mu \mathrm{m}$ motile unicellular alga. Rheological measurements performed on this system show a clear increase in effective viscosity when compared to a dead cell suspension. Shear thinning behaviour is also measured. Based on models elaborated for dilute suspensions, we provide an interpretation of our results, invoking the anisotropic distribution of live cell orientations within the shear flow. We then discuss two hypotheses that could account for the origin of this anisotropy: a gravity torque or an effective elongated aspect ratio due to flagella beating.

CR micro-algae 10] is a genus of green alga. It is a bi-flagellated unicellular organism. Chlamydomonas is used as a model organism for molecular biology, especially for studies of flagella motion, chloroplast dynamics, biogenesis and genetics. They are spheroidal in shape with two anterior flagella. Their back-and-forth movement produces a jerky breast stroke with a mean speed of $V \sim 40 \mu \mathrm{m} / \mathrm{s}$ in a water-like viscous medium. Since the cell radius is $R \sim 5 \mu m$, Brownian motion is negligible. The swimming direction of the cells can be directed by stimulus gradients: a phenomenon known as taxis, such as chemotaxis, rheotaxis or phototaxis. Gradients are not used in our experiments in order to avoid any external tropism on the motility. Wild-type strains were obtained from the IBPC lab in Paris [11]. Synchronous cultures of CR were grown in a Tris-Acetate Phosphate medium (TAP) using a $14 / 10 \mathrm{hr}$ light/dark cycle at $25^{\circ} \mathrm{C}$. Cultures were typically grown for two days under fluorescent lighting before cells were harvested for experiments. These cultures were concentrated up to a typical volume 

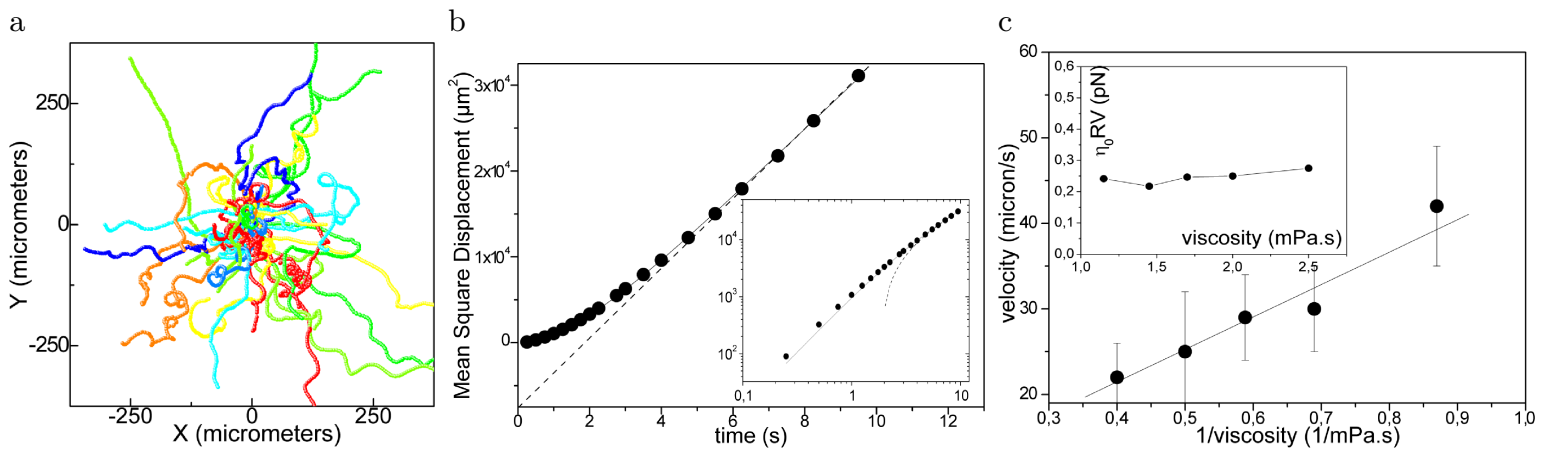

FIG. 1: a: Two dimensional trajectories of 50 swimming cells. Trajectory duration is 20 seconds. Trajectories starting positions have been all shifted to the origin. b: Measured mean square displacement (MSD) of cells as a function of time. Averages have been extracted from $9 \mathrm{~Hz}$ sequences of about 700 cells trajectories. MSD fits well to a persistent random walk process with a correlation time $t_{v}=3.5 \pm 0.1 \mathrm{~s}$ and a coefficient $D=995 \pm 20 \mathrm{\mu m}^{2} . \mathrm{s}^{-1}$ (see text). Inset: Log-Log plot. c: Measured mean velocity $V$ of cells as a function of the inversed viscosity of the medium. The line represents a linear fit. Inset: Viscous drag force should be proportional to the product of the medium viscosity $\eta_{0}$, the mean radius $R$ and the velocity $V$ of the cells. This product $\eta_{0} R V$ is shown to be independent of the medium viscosity. Error bars are smaller than the symbols.

fraction of 20 to $30 \%$ by centrifuging for 20 minutes at $900 \mathrm{~g}$ and re-suspending them in a fresh culture medium to achieve the volume fractions required. Volume fractions were measured using hematocrite capillaries and a Neubauer counting chamber.

We started by characterizing cell motility. Microscopy imaging of chlamydomonas suspensions was carried out on an Olympus inverted microscope coupled with a Sensicam camera used at frame rates up to $30 \mathrm{~Hz}$. Chambers made of glass and $240 \mu \mathrm{m}$ spacers were coated with bovine serum albumine in order to reduce cell adhesion. Cells were imaged in bright field using x10 magnification. Two dimension trajectories of cells were recorded, typically for a few tens of seconds. Particle tracking was performed using IDL [12]. The cell trajectories observed are correctly modelled by a persistent random walk [13, 14]. Short term correlations in the direction of movement are observed: the swimmer then presents an almost fixed direction during a characteristic time $t_{v}$. This short time stage corresponds to a ballistic regime characterized by a velocity $V$. The ballistic regime ends when swimmers make a turn. Note that the reorientation process has been studied recently by R. Goldstein [15] and is due to a dephasing of the two flagella for a short time. Hence the mean square displacement of cells is well described by a random walk behaviour with a persistence length $\ell_{p}$ of approximately ten cell diameters: $<x^{2}+y^{2}>=\ell_{p}^{2} t / t_{v}-0.5 \ell_{p}^{2}\left[1-\exp \left(-2 t / t_{v}\right)\right]$ where $<>$ represents an ensemble average over more than a thousand independent measurements [12]. For $t<<t_{v},<x^{2}+y^{2}>\sim V^{2} t^{2}$ with the ballistic velocity $V=\ell_{p} / t_{v}$ and for longer times a random walk is observed, $<x^{2}+y^{2}>\sim 4 D t$ with $D=\ell_{p}^{2} / 4 t_{v}$.

The algae velocity dependency on viscous drag was measured by adding a small amount of short chain dex$\operatorname{tran}(M w=20000)$ to the culture medium. This al- lowed variation of the viscosity of the medium between 1 and $2.5 \mathrm{mPa}$.s. We checked that swimming velocity is inversely proportional to bath viscosity $\eta_{0}$ (fig. 1). This suggests that the stall force (the force needed to stop the swimming cell), which is proportional to the product $\eta_{0} R V$, remains constant (fig. 1. c inset)

Let us now turn to the actual viscosity measurements. The effective viscosity of algae suspensions is measured on a Bohlin Gemini 150 rheometer equipped with coneplate geometry (cone angle $=2^{\circ}$, diameter $=60 \mathrm{~mm}$ ). Steady shear measurements were made at $T=20^{\circ} \mathrm{C}$. If a shear stress $\sigma$ is imposed and the associated shear rate $\dot{\gamma}$ measured, effective viscosity is $\eta_{\text {eff }}=\sigma / \dot{\gamma}$. Samples of different volume fractions were prepared and the effective viscosity measured for both live and dead cells. The cells were checked for motility after the rheometric measurement.

The effective viscosity $\eta_{\text {eff }}$ of active suspensions is found to decrease with the shear rate (fig. 2.a). As discussed below, it reveals competition between the shear rate and cells orientation in the flow. Note, that viscosity does not reach a plateau value. This suggests that other effects like segregation might play a role at higher shear rates. However, a clear global decrease of $\eta_{e f f}$ is observed from the maximum measured value at $\dot{\gamma} \approx 4 s^{-1}$. We then investigated the dependence of effective viscosity on the volume fraction of the suspension. To do so, viscosity was measured at a given shear rate of $5 s^{-1}$, which is sufficiently high for rheometer resolution but low enough to allow viscosity to be affected by motility.

Fig. 2. b shows the relative viscosity $\left(\eta_{\text {eff }}-\eta_{0}\right) / \eta_{0}$ of live and dead cell suspensions as a function of the volume fraction. In both cases, viscosity is an increasing function of the volume fraction as it is for passive beads. Remarkably, the effective viscosity of swimming cell suspensions is quantitatively larger than the viscosity of dead cell sus- 
a

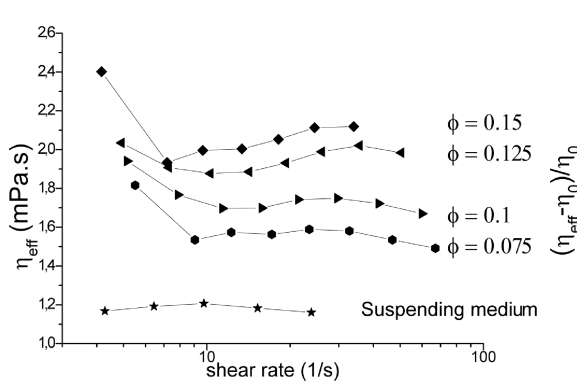

$\mathrm{b}$

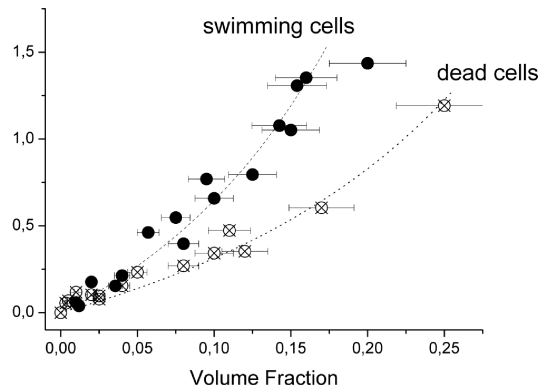

FIG. 2: a. Effective viscosity of chlamydomonas suspensions as a function of shear rate. Data are shown for different volume fractions of the suspension and star symbols represent the viscosity $\eta_{0}$ of the culture medium. The lines are shown for ease of viewing only. b. Relative viscosity of microswimmer suspensions (measured at shear rate $=5 s^{-1}$ ) as a function of volume fraction. Solid symbols represent live cell data and crossed symbols represent dead cell data. Measurements are fitted to equation 1 using $\phi_{\mathrm{m}}=0.62$ and $\alpha=2.5$ (dead cells) and 4.5 (swimming cells).

pensions (up to a factor of 2 for a $15 \%$ volume fraction).

The effective viscosity $\eta_{\text {eff }}$ of a suspension of passive spherical particles in a solvent of viscosity $\eta_{0}$, depends on its volume fraction $\phi$. Krieger and Dougherty's semiempirical law [16] is shown to provide a reliable description of the measurements:

$$
\eta_{\mathrm{eff}}=\eta_{0}\left(1-\frac{\phi}{\phi_{m}}\right)^{-\alpha \phi_{m}}
$$

Here, $\phi_{m}$ is the maximal packing volume fraction. For a dilute regime, where $\phi \ll 1$, eq. (11) reduces to $\eta_{\text {eff }} \approx \eta_{0}(1+\alpha \phi)$, where $\alpha$ is known as Einstein's intrinsic viscosity ( $\alpha=2.5$ for passive and spherical particles) 17.

To quantify the effect of motility on the effective viscosity of cell suspensions, we fitted the measurements using eq. (10). We set the maximal packing volume fraction to 0.62 and left $\alpha$ as a free parameter. This resulted in a large difference between active and passive suspensions. We found $\alpha=2.5 \pm 0.1$ in the case of dead cells, which clearly behave as passive particles. However, in the case of swimming cell suspensions, we obtained $\alpha=4.5 \pm 0.2$. Swimming cells induce a quantitative increase in the effective viscosity of the suspension.

In order to understand this increase in effective viscosity, we conducted complementary experiments which consisted of imaging the cells while subjected to a shear flow. Dead cells show a regular rotation at an angular velocity close to $\dot{\gamma} / 2$, as would passive spherical particles. We observed that swimming cells behave very differently: they resist the flow rotation for most of the time and eventually flip very rapidly. Fig. $3 \mathrm{~b}$ and b show picture sequences extracted from a fast-image film. High frequency acquisition $(500 \mathrm{~Hz})$ allows us to determine whether or not an alga is swimming by looking at the beating of flagella. In the case of a dead cell, flagella only move because of thermal agitation, whereas flagella of swimming cells beat at about $50 \mathrm{~Hz}$. Fig. 3 shows $50 \mathrm{~Hz}$ time sequences of cells subjected to a $10 s^{-1}$ shear rate. The swimming cell spends about two thirds of the period in the $x O y$-plane and flips during one third of the period whereas the dead cell rotates at a constant velocity close to $\dot{\gamma} / 2$.

Discussion. A puzzling question arises from our experiments: why should live cells and dead cells with the same aspect ratio and the same volume fraction respond so differently in a flow? In order to answer such a question, we propose some hypotheses based on existing models which might explain the increase of effective viscosity according to individual dynamics of live cells. As shown on fig. 1. b, the typical time $t_{v}$ after which a chlamydomonas changes its direction of motion is about 3 to 4 seconds. This is much longer than the typical time associated with the shear flow: $t_{f}=\dot{\gamma}^{-1} \approx 0.2 \mathrm{~s}$. This means that live algae resist the flow rotation (fig. 3-A) during approximately $70 \%$ of the time $t_{v}$ by being aligned in the flow. The fast flip between two aligned positions is indeed very fast in comparison to the time the cell spends parallel to the flow. After a time $t_{v}$, a reorientation occurs but the live alga is then aligned in the flow once again and so on. On the contrary, a dead cell rotates at a uniform angular velocity $\dot{\gamma} / 2$ like a passive particle.

As proposed by Ramaswamy [8] and Saintillan [7], when active particle distribution is anisotropic (i.e. aligned in the flow) it gives rise to a modified effective viscosity which is enhanced in the case of puller type cells (and reduced in the case of pusher type cells [9]). Following [7, 8] a swimming stress tensor can be defined: $\boldsymbol{\Sigma}^{s}=\sigma_{0}[<\mathbf{p} . \mathbf{p}>-\mathbb{I} / 3] . \sigma_{0}$ is the amplitude of the dipolar forces exerted by the cell to swim (for pullers $\sigma_{0}>0$ ). The vector $\mathbf{p}$ represents the orientation of the cell and in our case, it is oriented from the centre of each CR to the middle of the flagella anchor points. Active suspensions with $Q=<\mathbf{p . p}>-\mathbb{I} / 3 \neq 0$ are thus equivalent to an orientational ordered suspension with a non-zero deviatronic swimming stress contribution. This contribution to the effective shear viscosity is such that $\eta_{e f f}=\Sigma_{x y}^{s} / \dot{\gamma}$. 
Since for puller $\sigma_{0}>0$, effective viscosity is increased. These models although restricted to dilute regimes allow us to relate the observed cell alignment in flow to the resulting increase in viscosity.

We can think of two different hypotheses to explain the individual dynamics observed (fig 3, b): either gravity effects or large effective aspect ratio due to flagella beating. Gravity can exert a torque on cells since the centre of mass of $\mathrm{CR}$ is known not to coincide with its geometrical centre [2]. Ishikawa and Pedley [5] showed that a suspension of bottom heavy squirmers results in an increased effective viscosity. In 1970, Brenner [18] analytically predicted the effect of gravity on the rheology of a dilute suspension of inhomogeneous particles. He obtained for non rotating particles an intrinsic viscosity $\alpha=\frac{5}{2}+\frac{3}{2}$. This value is very close to our measurement of $\alpha$. Since dead cells are observed to tumble like passive spherical particles, this would suggest that mass distribution is homogeneous unlike live cells. However, we have no experimental evidence that this is the case. If the shear rate $\dot{\gamma}$ is increased, the constant torque $T_{G}$ exerted by gravity will eventually become smaller than the torque necessary to stop cell rotation $T_{F}=4 \pi \eta R^{3} \dot{\gamma}$. This would explain the shear thinning behaviour observed and also predicted by Brenner [18] in fig 2] a.

Another hypothesis that would explain the alignment of live cells is that their systematic flagella beating results in an effective hydrodynamic aspect ratio larger than that of their quasi-spherical shape [19]. The observed individual dynamics (fig 3) would then correspond to Jeffery's orbits of ellipsoidal prolate particles [20] that result in an increased effective viscosity. This is also predicted in [7, 8] where the puller suspension is close to a suspension of passive Brownian rods for which it is well known that a shear thinning behaviour can be observed [7] and [21]. Note that Brownian contribution is replaced here by hydrodynamic diffusivity due to hydrodynamic interactions between swimming CR.

In this paper, we have experimentally shown that active suspensions of puller type microswimmers present a dramatic increase in effective viscosity. We have correlated these macroscopic rheological measurements to the individual dynamics of cells under shear flow: swimming cells tend to resist the flow rotation unlike dead cells. More experiments are needed to explain the origin of this phenomenon: a gravity torque exerted on the cell or an effective large aspect ratio due to flagella beating.

We thank C. Nizak for his help in the choice of the system and C. Verdier for the rheometry. This work is financed by the Rhone-Alpes Region through the Cible program.

[1] X. Wu and A. Libchaber, Physical Review Letters 84, 3017 (2000).

[2] T. Pedley and J. Kessler, Annu. Rev. Fluid. Mech. 24, 313 (1992).

[3] N. Mendelson, A. Bourque, K. Wilkening, K. Andersoon, and J. Watkins, J. Bacteriol. 181, 600 (1999).

[4] C. Dombrowski, L. Cisneros, S. Chatkaew, R. E. Goldstein, and J. O. Kessler, Phys. Rev. Lett. 93, 098103 (2004).

[5] T. Ishikawa and T. J. Pedley, Journal of Fluid Mechanics 588, 399 (2007).

[6] B. Haines, I. Aranson, L. Berlyland, and D. Karpeev, Phys Biol 5, 9 (2008).

[7] D. Saintillan, Exp. Mech pp. DOI 10.1007/s11340-0099267-0 (2009).

[8] Y. Hatwalne, S. Ramaswamy, M. Rao, and R. A. Simha, Phys. Rev. Lett. 92, 118101 (2004).

[9] A. Sokolov and I. S. Aranson, Physical Review Letters 103, 148101 (pages 4) (2009).

[10] G. W. David Stern, Elizabeth Harris, ed., The Chlamydomonas Sourcebook (Academic, 2008).

[11] Physiologie Membranaire et Molculaire du Chloroplaste, UMR 7141, CNRS et Université Pierre et Marie Curie (Paris VI).

[12] J. Crocker and D. Grier, Journal of Colloid and Interface Science 179, 298 (1996).

[13] E. Codling, M. Plank, and S. Benhamou, Journal of the Royal Society Interface 5, 813 (2008).

[14] C. Patlak, Bulletin of Mathematical Biology 15, 311 (1953).

[15] M. Polin, I. Tuval, K. Drescher, J. P. Gollub, and R. E. Goldstein, Science 325, 487 (2009).

[16] I. M. Krieger and T. J. Dougherty, Trans. Soc. Rheol. 3, 137 (1959).

[17] A. Einstein, Ann. Phys. 19, 289 (1906).

[18] H. Brenner, J. of Colloid and Int. Science 32, 141 (1970).

[19] S. Ramaswamy, private communication: see http://www.condmatjournalclub.org/?p=760.

[20] G. Jeffery, Proceedings of the Royal Society of London 102, 161 (1922).

[21] E. Hinch and L. Leal, Journal of Fluid Mechanics 76, 187 (1976). 


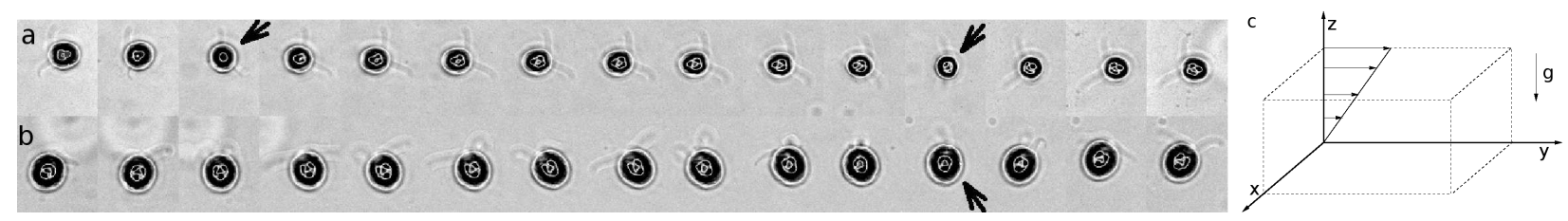

FIG. 3: A. Dead cell in a $10 \mathrm{~s}^{-1}$ shear flow experiencing tumbling around the $x$-axis. B. Live chlamydomonas swimming in the same shear flow. Arrows indicate cells flips and time between pictures is $20 \mathrm{~ms}$. The bar represents 10 micrometers. C. Schematic view of the flow cell, where $O z$ is the direction of observation, $y O z$ is the shear plane. 